\title{
Research on Anti-Duplication Technique of Security Printing for Office Documents
}

\author{
Jie Li \\ School of Information \\ Engineering, Beijing Institute of \\ Graphic Communication, \\ Beijing, China
}

\author{
Peng Cao \\ School of Information \\ Engineering, Beijing Institute of \\ Graphic Communication, \\ Beijing, China
}

\author{
Yuan Huang \\ School of Information \\ Engineering, Beijing Institute of \\ Graphic Communication, \\ Beijing, China
}

\begin{abstract}
This paper provides a halftone information hiding algorithm that can automatically identify whether office documents printed are an original or a hardcopy, and can be used to establish a safety guarantee for printed matter. By applying the method of 2ASK information modulation (binary amplitude shift keying), binarized anti-counterfeiting image-text is modulated as AM or FM halftone dots to record implanted watermark. Meanwhile, in order to ensure the watermark information in hiding effect of the original, optimal design is performed for AM and FM halftone dot patterns, their physical parameters (such as spatial arrangement and covering rate of halftone dot). Without the auxiliary condition of optical illusion background texture images, printing test and analyzing is designed for watermark images with various covering rates of halftone dot. This paper can solve the problems in security printing and copying, authentication and retrospection of documents printed such as official documents, legal instruments and certificates, and have application value in promoting the security of printing systems.
\end{abstract}

\section{Keywords}

Anti-counterfeiting, Security Printing, Halftone, Information Hiding, Anti-Duplication

\section{INTRODUCTION}

A kind of watermark image-text information that is hidden and invisible in the original but developing and visible in the copy can be implanted in the printed documents original to effectively distinguish the original and the copy, which can be particularly used to trace the source of the copy and plays an important role in the security of printing system.

Nowadays, the research on anti-copying printing technology can be carried out mainly in processing and image. The former based on the processing includes anti-copying materials [1,2,3], special inks[4,5,6] and special devices [7], etc. and the latter based on the image achieves the purpose of anti-copying through certain graphic design, watermark[8,9,10], etc. In terms of anti-copying effect, the original cannot be photocopied and there is an obvious distinction between the original and the copy. The special pattern latent image technology, a kind of proven technology, can apply the AM and FM halftone dots image information to realize that the original is invisible but the copy is visible, which mainly makes use of photocopier's insufficient ability to recognize different network lines and different halftone dots to realize the image anti-copying. In other words, a layer of designed shading that is composed of the latent image formed by FM halftone dot and the carrier image formed by AM halftone dot, can be printed on the paper before the documents are printed and have good effect in anti-copying.
Compared with the partial anti-copying by other technologies or the hidden effects by special patterns, it is difficult to identify the encrypted information in the original on account of no special patterns, but the hidden encrypted information is clearly found in the copy result from the latent image hiding and overspreading in the shading of documents. Furthermore, this technology has the advantages of good anti-copy effect, low cost, less reliance on hardware equipment and so on.

\section{RELATED WORD}

\subsection{AM/FM Hybrid Network Information}

\section{Hiding Technology}

The use of microstructure technologies, such as halftone dots, the fine mesh lines and the micro prints[11,12,13] for anticounterfeiting has become more common. This kind of method does not need the use of special printing materials or a special printing method thereby reducing the cost of manufacture. The watermark pattern is hidden in the micro-structure of different sizes of halftone dots that exceeds the discernible ability of human visual system. Beyond a certain distance, the information or patterns hidden using this technology cannot be recognized by the human eye. However, the originally hidden informa-tion or patterns will be displayed after the duplicator makes the insufficient sampling of halftone-dot microstructures. This mechanism can avoid using the copier to make forged or unauthorized copies.

The watermark patterns that are composed of AM and FM halftone dots can not be perceived by the human eye beyond a certain distance. This is done through outputting the densitycalibration chart to choose the proper density parameters, which are applied to the manufacture process of watermark with hybrid halftone dots. The hidden patterns of a water-mark need to be preprocessed first, and thereby making the image blocks of AM and FM halftone dots to be combined smoothly. The copy machine, the lenticular lens and the optical scanner can detect the hidden watermark in the printed shadow image[14].

The above-mentioned density calibration includes making a density-calibration chart. Take the density of AM halftone dot as the background to output the FM halftone dots within a certain range of density, then the human eye or a video camera recognizes the best matching parameters that have the optimal hidden effect.

\subsection{Optical Illusion Auxiliary Image}

In order to solve the problem that the watermark information is difficult to hide due to the quantization error of AM dots and FM dots in the same grayscale in the halftone watermark image composed of AM/FM mixed dots, the optical illusion assists the 
$\mathrm{AF} / \mathrm{FM}$ mixed dots under the condition of the image Information hiding technology is proposed [15].

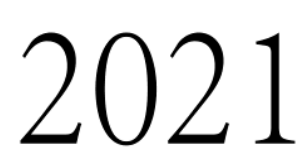

(a)

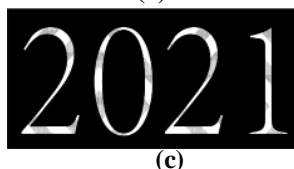

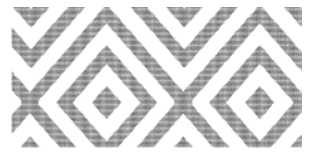

(b)

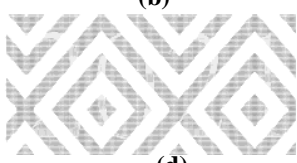

(d)
Figure 1. : Optical illusion auxiliary image. (a) Watermark image. (b) Halftone optical illusion auxiliary image. (c) Halftone watermark image. (d) Encrypted image with watermark.

As shown in Figure 1, by using the hybrid network method, the embedding and extracting of the encrypted information can be realized effectively, and can realize the anti-copy of the image. Generate two masks to overlay, can better enable encryption information fusion and shading pattern in the picture, at the edge of the stack after processing can be perfect to achieve a better visual effect.

\subsection{ASK Modulation Technology}

Binary amplitude keying is to key a continuous carrier with a baseband rectangular pulse representing a binary digital signal. When there is carrier output, it means sending " 1 ", and when there is no carrier output, it means sending " 0 ". According to the principle of digital image modulation, the 2ASK signal is generated using the on-off keying method:

$$
\begin{aligned}
G_{0}=\sum_{i=1}^{n} \sum_{j=1}^{m}\left(G_{w}(i, j) \cdot G_{1}(i, j)\right. \\
\left.+\overline{G_{w}(i, j)} \cdot G_{2}(i, j)\right)
\end{aligned}
$$

Where $G_{0}$ represents the combined halftone image, $G_{\mathrm{w}}$ is a bilevel hidden-pattern mask, $G_{1}$ represents the image of AM halftone dot, and $G_{2}$ represents the image of FM halftone dot.

\section{AM/FM NETWORK PATTERN SETTING}

The design of threshold matrix, the core and focus of this algorithm, aims to control the size and position of ink dots to achieve the integration of AM and FM halftone dots.

On account of quantization errors, AM and FM screening under the same gray-scale will lead to the integrated halftone dots and have an obvious visual visibility. Therefore, it is more important to do the gray-scale matching test. Compared with the AM screening image with the specified gray scale (taking as a comparison chart ) and a series of FM screening image with similar gray scales, the best halftone dot matching will be selected and determined by subjective and objective judgments.

The subjective judgment shows that the best parameter or parameters will be selected by the visual inspection in accordance with the gray-scale fusion degree after the testing strips is designed and printed. And afterwards, when one or more parameter is diffused and the testing space is narrowed, the selection will be repeated until there is no choice by the visual inspection.
As for the objective judgment, it needs to photograph and upload the printed testing strip and then randomly select the multiple lines for the gray-scale broken line graph or the histogram. The smaller the gray-scale error is, the smaller the curve fluctuation it has. The higher solid smoothness refers to the better matching of parameters. As shown in Figure 2, it is tested under the circumstance of $14 \%$. Significantly, there is a certain error between the electronic testing strip and the actual printed result, which is also the theoretical basis for this experiment. In the histogram, a row of pixels represents the grayscale testing strip and fluctuates up and down with the change of position, while the junction of AM and FM halftone dots fluctuates largely and demonstrates the integration effect with this parameter.

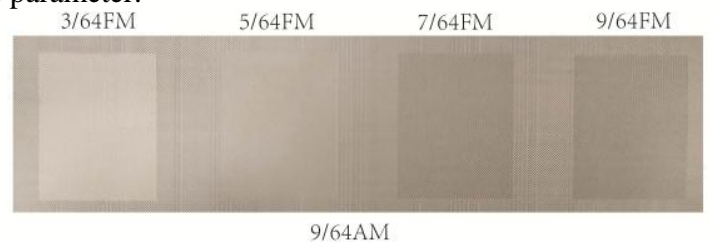

(a)Gray test strip design

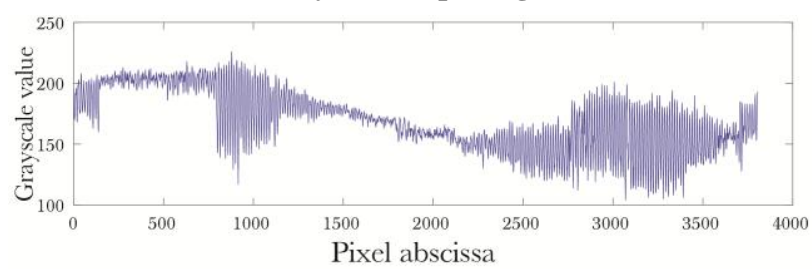

(b)Gray histogram

Figure 2.: Gray matching test(14\%)

The specific design algorithm is as follows:

- Determine the grayscale value, and set the grayscale control below $20 \%$.

- Set the initial AM dot pattern and a series of similar grayscale FM patterns according to the grayscale.

- Combine multiple FM patterns and one AM pattern in a certain way to make a subjective judgment test strip and printout.

- It is judged by the human eye which FM network and AM network are compatible with each other in the printed result, then the best one is selected, and if it does not meet the conditions, the pattern is redesigned.

- The pattern that meets the conditions will be selected for objective judgment, and the pattern will be redesigned when the curve fluctuates too sharply than expected.

- Repeat the above process until the best AM/FM dot pattern is obtained, and then set the screening template according to the pattern.

Assuming that the selected gray level is $x$ and the adapted function is $f(x)$, the gray level that the adapted FM network should be set to be $y$ :

$$
y=f(x)=f_{1}(x) \cdot f_{2}(x)
$$

Where, $f_{1}(x)$ is function of point gain, $f_{2}(x)$ is function of mechanical correction. Numerical comparison of area rates of the halftone network is obtained, as shown in Table 1.

According to the above parameters, set the halftone template to achieve the above dot coverage ratio. The principle of screening

Table1.: Dot coverage parameter setting

\begin{tabular}{ccc}
\hline AM dot & \multicolumn{2}{c}{ FM dot coverage parameter } \\
\cline { 2 - 3 } coverage & inkjet printing & laser printing \\
\hline
\end{tabular}




\begin{tabular}{lcc}
\hline $3.1 \%$ & $2.3 \%$ & $2.3 \%$ \\
$7.0 \%$ & $6.6 \%$ & $5.0 \%$ \\
$10.0 \%$ & $8.9 \%$ & $7.4 \%$ \\
$14.1 \%$ & $12.0 \%$ & $10.5 \%$ \\
\hline
\end{tabular}

template setting is that the overall center of mass of the scattered pixels of the FM dots is close to the corresponding AM dots in space.

The halftone template design is shown in Figure 3.

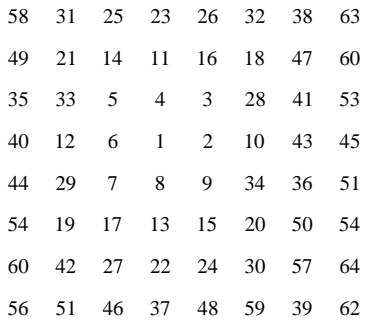

(a) (b)

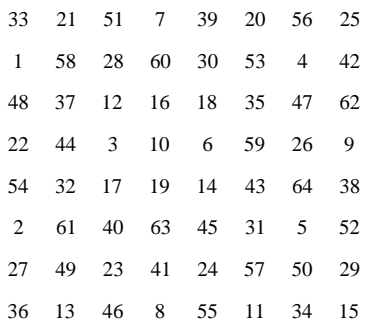

Figure 3.: Halftone template (a)AM template; (b)FM template

It can be seen that the pixel points of the two screening methods must have the best number ratio, and the corresponding local areas need to have the same or close centroids, and the frequency modulation network should be relatively evenly distributed as much as possible because of the gain effect of printing.

\section{WATERMARK EMBEDDING ALGORITHM}

The printed document is embedded with variable or immutable graphic information in a visually invisible form, and is printed together on the substrate. The specific process is shown in Figure 4.

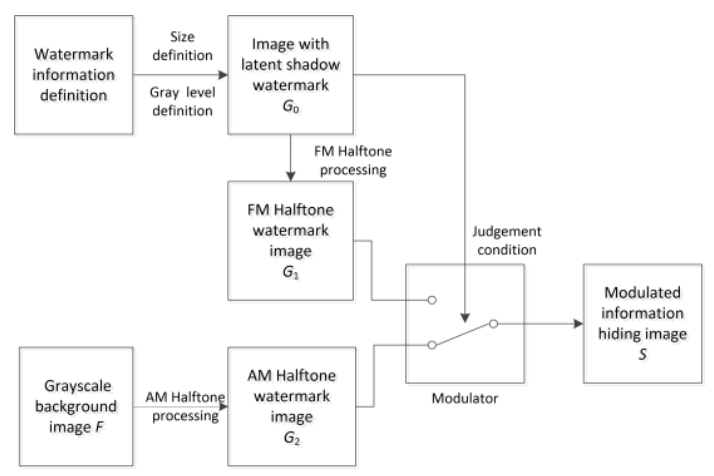

Figure 4.: Algorithm flow chart

When the printed manuscript is scanned and copied, the invisible graphic watermark information hidden in it will be developed, which can be used for anti-counterfeiting or copy identification and traceability of the printed manuscript.

\subsection{Definition Of Watermark Information And Information Modulation}

Logo, text, or other warning information can all be watermark information, and the area and shape of its format can be designed according to requirements.

Assuming that the required grayscale is $x$, halftone processing is performed on the background image of $x$ grayscale using AM screen-ing, and then halftone processing is performed on the watermark part of the grayscale $k x$ obtained by the correction parameter k using FM screening. This article uses the halftone template designed in the third section to screen to control the position and size of the ink dots. Assuming that the FM network template is $T_{1}$ and the AM network template is $T_{2}$. The generation of FM halftone watermark image and AM halftone watermark image satisfies:

$$
\begin{aligned}
& G_{1}(i, j)=\left\{\begin{array}{lcc}
1, & \text { if } & k G_{0}(i, j) \leq T_{1}(i, j) \\
0, & \text { others }
\end{array}\right. \\
& G_{2}(i, j)=\left\{\begin{array}{ccc}
1, & \text { if } & F(i, j) \leq T_{2}(i, j) \\
0, & \text { others }
\end{array}\right.
\end{aligned}
$$

Where $G_{1}$ is AM halftone image to be water-marked, when the corresponding AM matrix point is 1 , fill the position with a black point, otherwise it is a white point, $G_{2}$ is $F M$ halftone image to be water-marked, when the corresponding FM matrix point is 1 , fill the position with a black point, otherwise it is a white point, $k$ is correction parameters.

Table 2 shows the steps of the lookup table algorithm. In addition to the 2ASK modulation, the hidden information image generation can also be generated by the modulation method of the look-up table, and the corresponding scheme can be selected according to different situations. Reduce the time complexity of the algorithm at the cost of reducing part of the image quality.

\begin{tabular}{|c|c|}
\hline steps & Algorithm content \\
\hline Input & Watermark image $G_{0}$ \\
\hline Output & Information hiding image $S$ \\
\hline Step.1 & Reading watermark image $G_{0}$ \\
\hline Step. 2 & $S \longleftarrow[]$, image $1 \longleftarrow[]$, image $2 \longleftarrow[]$ \\
\hline Step. 3 & for $i=1$ to $n d o$ \\
\hline Step.4 & for $j=1$ to $m$ do \\
\hline Step.5 & if $G_{0}(i, j)$ is the watermark area \\
\hline Step.6 & $\begin{array}{l}\text { image } 1(i, j) \text { set as black dots, } \\
\text { image } 2(i, j) \text { set as white dots; }\end{array}$ \\
\hline Step.7 & if $G_{0}(i, j)$ is the non-watermark area \\
\hline Step.8 & $\begin{array}{l}\text { image } 1(i, j) \text { set as white dots, } \\
\text { image } 2(i, j) \text { set as black dots; }\end{array}$ \\
\hline Step.9 & end;end; \\
\hline Step.10 & $G_{1} \longleftarrow$ image 1 AM halftone precessing \\
\hline Step.11 & $G_{2} \longleftarrow$ image 2 FM halftone precessing \\
\hline Step. 12 & $S \longleftarrow G_{1}+G_{2}$ \\
\hline Return $S$ & \\
\hline
\end{tabular}

Table2. : Lookup Table Algorithm Steps

\subsection{Printout And Results}

Use two mainstream digital printing meth-ods to printing: inkjet printing (HP Indigo6900) printing machine) and laser printing ("RICOH Pro C7100X" printing machine). To get the desired result, the printer's print settings need to match the saved print file. Among them, the key setting is that the resolution is above $600 \mathrm{dpi}$,and RIP with the highest number of lines can preserve the dot details to the greatest extent. Figure 6 shows the actual effect of laser printing. FM/AM dots can be smoothly transitioned and relatively integrated, as well as it will not affect the information display effect of the document content 
Table 3 and Table 4 show the actual printing results of the two printing methods. It can be seen that all the experimental drafts meet the requirements, that is, the hidden watermark information cannot be recognized beyond a certain distance. However, except for the first group's overall dot fusion rate is sufficiently high, other groups can still see some traces at close range, which will be further processed later.

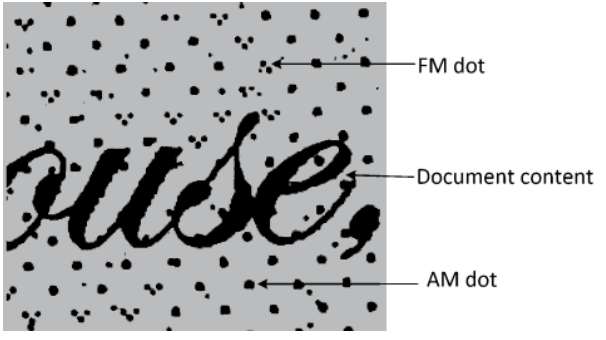

Figure 5. : Printout image(laser printing,3.1\%)

Table 3.: Printout image(laser printing)

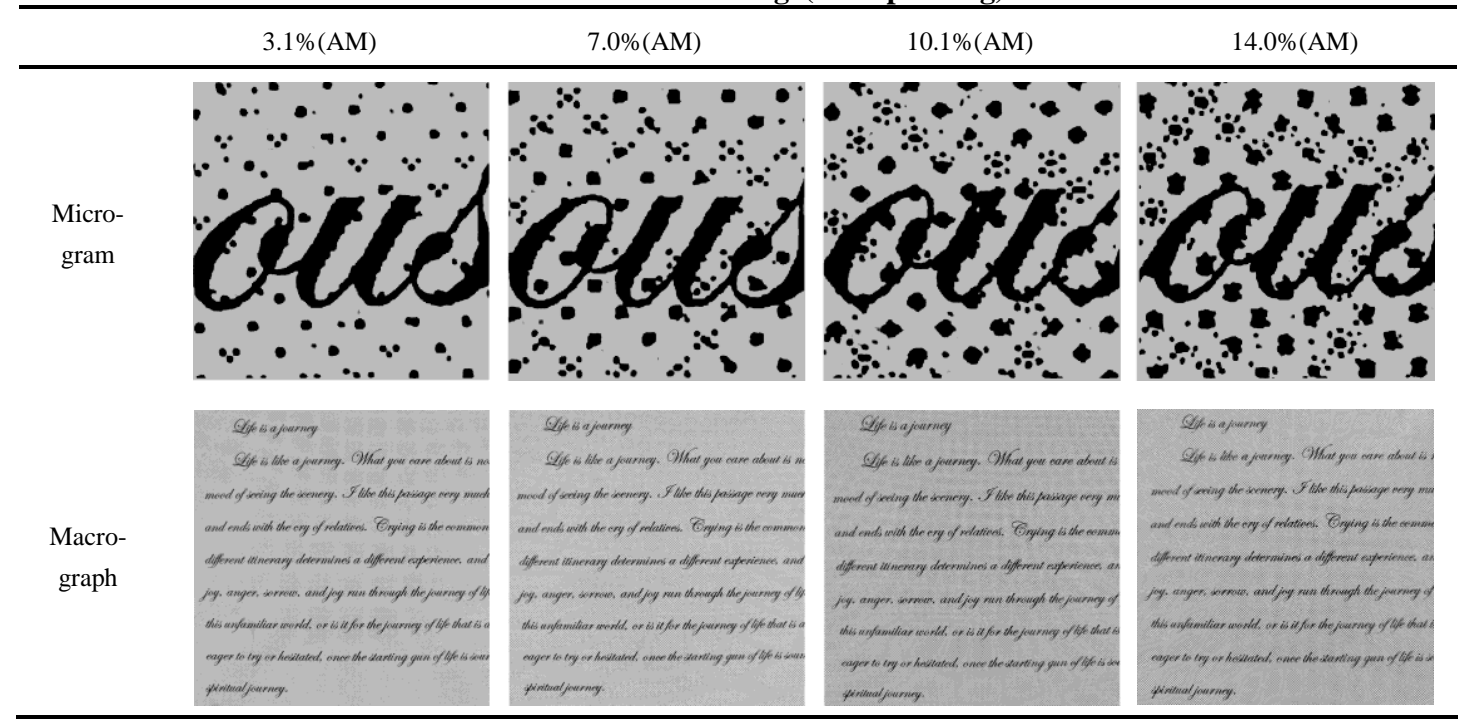

Table 4.: Printout iamge(inkjet printing)

Micro-
Macro-
graph

The copy effect of the document is an important step to verify the anti-counterfeiting characteristics of this algorithm. This article tested the copying effects of a variety of copiers, and the results showed that the watermark information can be easily recognized by human eyes. Among them, the copy result of the FUJI XEROX copier is shown in Table 5. It can be seen that the displayed warning information can be seen under normal viewing distance. 
Table 5.: Duplicate image(600dpi)

- $3.1 \%(\mathrm{AM})$ 7.0\%(AM)

The reason why hidden watermarks are obvious in close view at some gray levels is that the quantization error of the two screening methods has not been eliminated. In fact, in the case of 600dpi, finer adjustments cannot be made, and the randomness and uncontrollability of mechanical printing dot will also cause certain errors. In the end, the introduction of noise became a better choice. In this experiment, the noise is salt and pepper noise, and a certain amount of salt and pepper noise is introduced into the shading image, which successfully reduces the recognition of the quantification error of the two screening methods by the human eye, and at the same time reduces the discrimination between the original and the duplicate to a certain extent. The method of adding noise points is shown in Table 6 .

\begin{tabular}{ccc}
\multicolumn{2}{c}{ Table 6.: } & Noise addition parameter setting \\
\hline \multirow{2}{*}{$\begin{array}{c}\text { Gray } \\
\text { level(AM) }\end{array}$} & \multicolumn{2}{c}{$\begin{array}{c}\text { Parameter(Percentage of total pixels of } \\
\text { carrier image) }\end{array}$} \\
\cline { 2 - 3 } & Hp Indigo6900 & Ricoh Pro C7100X \\
\hline $7.0 \%$ & $0.020 \%$ & $0.025 \%$ \\
$10.1 \%$ & $0.015 \%$ & $0.015 \%$ \\
$14.0 \%$ & $0.010 \%$ & $0.005 \%$ \\
\hline
\end{tabular}

As the gray level increases, the number of added noise points decreases; otherwise, the watermark visibility of the duplicate will be greatly affected.

As shown in figure 7, the addition of noise increases the anticounterfeiting performance of the algorithm, which increases the disorder of the image, causes certain visual confusion, and plays a certain protective role in the watermark image.

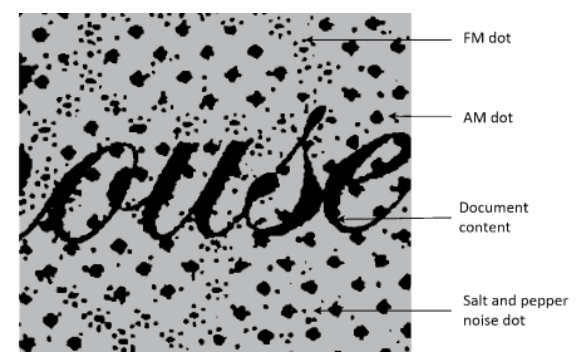

Figure 6.: Printout image with noise(laser printing,10.1\%)
Table 7 and Table 8 show the printing effect of the image with noise under laser printing and inkjet printing. Even if you look at it from a close distance, you can hardly see the marks of the watermark, and the watermark hiding effect is good.

Table 9 shows the copy effect after adding noise points, indicating that the display effect of the photocopy watermark is not significantly weakened, and the warning information can be clearly and completely displayed. In particular, the photocopying effect is better when the watermark information is spread all over the document. It has a good anticounterfeiting capability and certain document traceability ability.

Table 7.: Printout image with noise(laser printing)

level(AM)


Table 8.: Printout image with noise(inkjet printing)

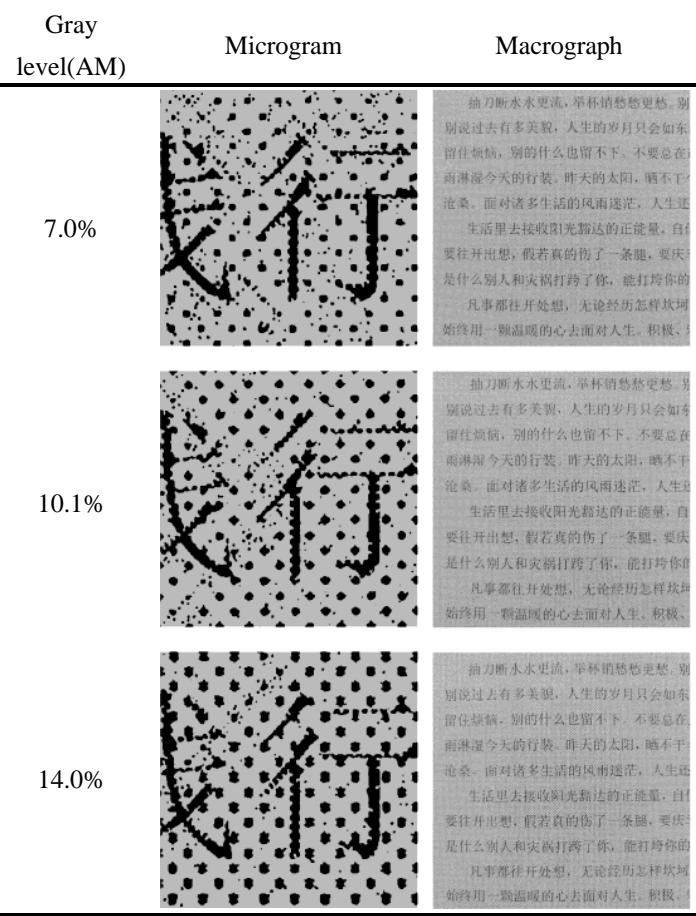

Table9.: Noisy duplicate image(600dpi)

Gray
level(AM) laser printing inkjet printing

\section{CONCLUSION}

In this article, the AM and FM screening technology are used to hide and overspread the watermark information in the shading, clearly distinguishing the copy and the original without affecting the content of documents. To reduce the quantization errors for two methods of screening, the methods of adjusting the number and position of FM halftone dots, using small AM halftone dots as the core to drive several FM halftone dots, bringing in image noise and others are provided to lower the recognition of latent image with human eyes in the original, playing a certain role in anti-counterfeiting performance. Therein lies the ratio of the number of AM black pixels to the number of FM black pixels requiring the actual coverage areas of AM or FM according to the physical resolution of 600dpi. To achieve or get close to the ideal ratio, it can increase or decrease the number of FM pixels on the adjacent units. If the smaller AM halftone dot is taken as the core, the aggregated pixels should be less than half of the total. The final result is that a piece of ordinary paper is covered with a thin layer of ink but is overspread with the hidden latent image information. There is no clear distinction between the original and the copy for the visual inspection unless the document printed normally is photocopied. Relying on the evident watermark information hidden in the shading, it is easy to distinguish the original and the copy and realize the function of anti-counterfeiting. Aiming at different printing methods and different printing devices, the density of halftone dots, namely the arrangements, varies from AM and FM adaptive curves for best effect. Furthermore, special devices, inks or papers become unnecessary except a layer of thin ink that should be printed in advance at no additional printing cost. As the physical resolution increases, a good visual effect on the original without image noise can come true for the follow-up. If all the gray-scales have corresponding parameters, the information can be hidden directly in the grayscale image or even a large amount of information can be hidden in the color image.

\section{ACKNOWLEDGMENTS}

This work was supported by National Natural Science Foundation of China (61972042), Beijing Fund-Municipal Education Commission Joint Project (KZ202010015023) and Research Project of Beijing Institute of Graphic Communication (Ef202001).

\section{REFERENCES}

[1] 3DTL, Inc. 2017. Anti-copy optical materials and methods. US: US9658373B2

[2] A Kaminska. 2019. Don't copy that: Security printing and the making of high-tech paper. The InternationalJournal of Research into New Media Technologies. 25(4), 590606.

[3] Wang X, Chen X, Xu G, Li J, Guo J, Wang Q. 2021. Performance of zinc oxide quantum dots coated paper and application of fluorescent anti-counterfeiting. Applied optics. 60(8), $2304-2313$

[4] Wan Zhang,Xian fu Wei, Bei qing Huang, Chun yan Lu. 2011. The Effect of Resin on the Property of Red Fluorescent Inkjet Ink. In Proceedings of International Conference on Digital Printing.

[5] X Chen,Q Wang,XJ Wang,J Li,GB Xu.2021. Synthesis and performance of $\mathrm{ZnO}$ quantum dots water-based fluorescent ink for anti-counterfeiting applications. Scientific Reports. 11(1), 5841-5841.

[6] A Goyat, N Singh. 2016. Currency Printing and Various Security Design Features. International Journal of Science. 6(3),248-250.

[7] Szu Chi LO. 2016. Anti-copy electronic device. US: US10037442B2.

[8] Prabhjot Kaur Chahal, Jasveen Kaur, Palwinder Sngh. 2014. Digital Watermarking On Bank Note. International Journal of Soft Computing and Engineering. 3(6), 38-41.

[9] Renita Maria Colaco, Riona Fernandes, Reenaz, Sowmya S. 2021. Efficient Image Processing Technique for Authentication of Indian Paper Currency. In Proceedings 
of 2021 International Conference on Computer Communication and Informatics.

[10] A Upadhyaya, V Shokeen. 2018. Counterfeit currency detection techniques. In Proceedings of the 8th International Conference on Cloud Computing, Data Science \& Engineering.

[11] GN Simonian. 2013. Taking the multifaceted approach: security printing. African Printer. 1,12-13.

[12] Miao Quanlong, Zhang Yixin, Tang Cheng, Tang Mei. 2014. Multi-channel optical anti-counterfeiting of micromirror arrays. Optik. 125(1), 583-586.
[13] Ambadiyil Sajan, Jayan K.G., Prabhu Radhakrishna, Mahadevan Pillai V.P. 2015. Micr-ostructure encryption and decryption techniques in optical variable and invariable devices in printed documents for security and forensic applications. In Proceedings of the Optical Sensors.

[14] Hsi-Chun Wang, Hsiao and Chi-Ming Lien.2006. Method of watermark with hybrid halftone dots. US: No.0170974.

[15] Chen Fangfang, Cao Peng, Zhu Jianle and Huo Peijun. 2017. Print Anti-replication Technology Based on AM/FM Hybrid Halftone. In Proceedings of the 17th IEEE International Conference on Communication Technology. 